\title{
Blood carnitine profiling on tandem mass spectrometry in liver cirrhotic patients
}

\author{
Hisamitsu Miyaaki ${ }^{1 *}$, Hironori Kobayashi ${ }^{2}$, Satoshi Miuma', Masanori Fukusima'', Ryu Sasaki ${ }^{1}$, \\ Masafumi Haraguchi ${ }^{1}$ and Kazuhiko Nakao ${ }^{1}$
}

\begin{abstract}
Background: The level and profiles of blood free carnitine and acylcarnitines, obtained by acylcarnitine analysis using tandem mass spectrometry, reflect various metabolic conditions. We aimed to examine the level of free carnitine and acylcarnitines in liver cirrhosis patients by acylcarnitine analysis and determine the clinical and subjective factors associated with blood carnitine fraction levels in liver cirrhosis.
\end{abstract}

Methods: We compared blood carnitine fractions in 54 liver cirrhotic patients to other laboratory test results and questionnaire answers.

Results: In almost all patients, the blood levels of free carnitine (C0) and acetylcarnitine (C2) were within the normal reference range. However, in some patients, the levels of long-chain acylcarnitines, such as C16 and C18:1acylcarnitine, were higher than the normal reference range. Liver function, assessed by Child-Pugh score, was significantly correlated with the blood level of each carnitine fraction measured $(C 0, C 2, C 3, C 4, C 6, C 10, C 12, C 12: 1$, C14:1, C16, C18:1, and C18:2-acylcarnitine). Cirrhotic symptom score was significantly correlated with C0, C2, C3, C16, and C18-1-acylcarnitine blood levels. Among the 36-item short-form health survey (SF-36) items, the physical component summary was significantly associated with C0, C2, and C18-1-acylcarnitine blood levels.

Conclusions: Carnitine fraction levels were positively correlated with liver cirrhosis stage, particularly, long-chain acylcarnitines. Moreover, carnitine fraction levels were associated with various subjective physical symptoms in liver cirrhosis patients.

Keywords: Carnitine fraction, Liver cirrhosis, Acylcarnitine, Tandem mass spectrometry

\section{Background}

Carnitine is known to be an important biofactor in fatty acid oxidation. Carnitine is essential for the transport of long-chain fatty acids from cytoplasm to mitochondria. The acylcarnitine in which an acyl-base derived from long-chain fatty acid and carnitine are ester-linked is transported into mitochondria, where acylcarnitines are converted to acyl$\mathrm{CoA}$ at the inner mitochondrial membrane, and are provided as the substrate for $\beta$-oxidation. Moreover, it eliminates intracellular acyl-compounds, regulating the ratio of coenzyme A (CoA) to acyl-CoA $[1,2]$.

Blood carnitine level could be influenced by various metabolic states, such as liver cirrhosis, end stage renal failure,

\footnotetext{
* Correspondence: miyaaki-hi@umin.ac.jp

${ }^{1}$ Department of Gastroenterology and Hepatology, Nagasaki University Graduate School of Biomedical Sciences, 1-7-1 Sakamoto, Nagasaki 852-8501, Japan

Full list of author information is available at the end of the article
}

and type 2 diabetes [3-6]. Administration of L-carnitine is used for the treatment of cirrhosis-related symptoms, such as muscle cramps and hepatic encephalopathy $[4,7]$.

Acylcarnitine analysis using tandem mass spectrometry (MS/MS) has been developed as a test for fatty acid oxidation disorders, congenital defect in mitochondrial fatty acid oxidation enzymes, in pediatric field.

Carnitine exists as free carnitine and acylcarnitine fractions in blood samples; the acylcarnitine fraction can be further subdivided into multiple compounds. The amount of each acylcarnitine fraction in a blood sample was too small to accurately measure until recently. Advances in technologies, such as MS/MS, have enabled detailed analyses of acylcarnitine fractions in blood; however, there have been few studies on the factors associated with blood acylcarnitines in patients with liver cirrhosis [8]. 
In this study, we measured the free carnitine and acylcarnitines in patients with liver cirrhosis using MS/MS and compared the results with data from other laboratory tests and questionnaires.

\section{Methods}

Study population, design, and ethics statement

Fifty-four patients (median 67 years; male:female 34:20) with liver cirrhosis being treated at Nagasaki University Hospital were enrolled in this study. Twenty-four patients had hepatocellular carcinoma (HCC). We diagnosed liver cirrhosis based on clinical and laboratory data or histologic examination of liver biopsy specimens. Patients' clinical characteristic are shown in Table 1.

Six patients received a protein diet restriction (range 30 to $50 \mathrm{~g}$ daily) and 12 patients received a sodium restricted diet (6 mg daily).

Free carnitine $(\mathrm{C} 0)$ and each acylcarnitines (i.e. $\mathrm{C} 2$, C4, C5, C6, C8, C8:1, C10, C12, C12:1, C14:1, C16, C18, C18:1, and C18:2) in a dried blood spot (DBS) were measured by using dried blood spotDBS with MS/MS in accordance with standardized protocols of non-derivatized method using NeoBase kit (Perkin Elmer, MA, USA). In brief, a single $3 \mathrm{~mm}$ DBS punch was placed in each well of 96-well assay plate, and $100 \mu \mathrm{L}$ of the extraction solution containing internal standard of acylcarnitines and amino acids was added to each well. The plate was shaken at $700 \mathrm{rpm}$ at $45^{\circ} \mathrm{C}$ for $45 \mathrm{~min}$, and the supernatant was transferred to another plate after the plate was centrifugation for $5 \mathrm{~min}$ at $1000 \times \mathrm{g}$. MS/MS analyses was performed by flow injection. Samples were measured using a Nexera MP System utilizing the SIL30ACMP Multi-Plate autosampler and LCMS-8040 triple quadrupole mass spectrometer (Shimadzu Corporation, Kyoto, Japan) with an ESI positive source. The mobile phase was provided using the NeoBase Kit. The main component of the mobile phase was methanol. Injection volume was $1 \mu \mathrm{L}$, and total analytical time was 1 min.

The measurements were evaluated by using reference values optimized for clinical testing. The levels of free

Table 1 Patients' clinical characteristics $(n=54)$

\begin{tabular}{ll}
\hline Age, years (median, range) & $67(32-84)$ \\
\hline Gender, male/female & $34 / 20$ \\
Child-Pugh score & $7(5-14)$ \\
Body mass index $\left(\mathrm{kg} / \mathrm{m}^{2}\right)$ & 23.5 \\
Etiology HBV/HCV/AH/NASH/others & $5 / 14 / 17 / 6 / 13$ \\
HCC +/- & $24 / 30$ \\
eGFR & $68.0(34-126)$
\end{tabular}

Abbreviations: $H B V$ hepatitis $B$ virus; $H C V$ hepatitis $C$ virus; $A H$ alcoholic hepatitis; NASH non-alcoholic steatohepatitis; HCC hepatocellular carcinoma; eGFR estimated glomerular filtration rate carnitine and acylcarnitines were assessed correlation of clinical factors, such as age, gender, body mass index (BMI), blood ammonia level, Child-Pugh score, HCC, and estimated glomerular filtration rate (eGFR), which were previously reported to be correlated with carnitine levels $[6,8]$.

Written informed consent was obtained from all patients. This study was approved by the ethics committee of Nagasaki University (No, 17022735).

\section{Questionnaires}

The cirrhosis-related symptom scores (CSS) questionnaire, which we developed, was used to evaluate cirrhosis symptoms [9]. The Epworth Sleepiness Scale (ESS) was used to evaluate daytime hypersomnolence [10]. Sleep quality was assessed using the Japanese version of the Pittsburgh Sleep Quality Index (PSQI) [11]. Healthrelated quality of life was evaluated using the Japanese 36-item short-form health survey (SF-36) [version 2; Medical Outcomes Trust (Hanover, NH, USA), Health Lab (Hanover, NH, USA), QualityMetric (Lincoln, RI, USA), and Shunichi Fukuhara (iHope International; Kyoto, Japan)].

\section{Statistical analysis}

We analyzed all data using SPSS version 20.0 software (SPSS, Chicago, IL, USA) and $P<0.05$ was considered statistically significant. Discrete variables are presented as means with ranges. Laboratory data were analyzed using t-tests or chi-square tests, as appropriate. Correlations were determined using Pearson's linear regression analysis. A multivariate analysis was performed using binary logistic regression analysis.

\section{Results}

Acylcarnitine analyses in cirrhotic patients

Average levels of free carnitine $(\mathrm{CO})$ and acylcarnitines in dried blood spot of cirrhotic patients are shown in Table 2.

In most cirrhotic patients, C0 (46 patients) and C2 (51 patients) values were within the normal range. Some patients had C16 (10 patients) and C18-1 (23 patients) levels higher than the normal range. The Child-Pugh score was significantly correlated with blood levels of $\mathrm{C} 0, \mathrm{C} 2, \mathrm{C} 3$, C4, C6, C10, C12, C12:1, C14:1, C16, C18:1, and C18:2 (Table 3). C18:1 level was high in all patients with a ChildPugh score of more than 11 (Fig. 1a, b, c, d). Blood ammonia level was significantly correlated with $\mathrm{C} 0(\mathrm{r}=0.363$, $p=0.011)$ and C8 levels $(\mathrm{r}=0.355, p=0.014)$.

\section{Factors associated with acylcarnitines}

The acylcarnitines found in the highest quantities in blood (i.e. C0, C2, C3, C16, and C18-1) were analyzed for associations with other clinical parameters. In 
Table 2 Carnitine fraction levels (mean \pm s.d.) (Standard values)

\begin{tabular}{ll}
\hline C0 (Free carnitine) $\mathrm{mmol} / \mathrm{L}$ & $50.4 \pm 20.8(20 \sim 70)$ \\
\hline C2-acylcarnitine $\mathrm{mmol} / \mathrm{L}$ & $24.1 \pm 12.1(5 \sim 45)$ \\
C3-acylcarnitine $\mathrm{mmol} / \mathrm{L}$ & $3.1 \pm 1.7(\sim 3.5)$ \\
C4-acylcarnitine $\mathrm{mmol} / \mathrm{L}$ & $0.23 \pm 0.11(\sim 1.4)$ \\
C5-acylcarnitine $\mathrm{mmol} / \mathrm{L}$ & $0.25 \pm 0.67(\sim 0.7)$ \\
C6-acylcarnitine $\mathrm{mmol} / \mathrm{L}$ & $0.10 \pm 0.04(\sim 0.15)$ \\
C8-acylcarnitine $\mathrm{mmol} / \mathrm{L}$ & $0.10 \pm 0.05(\sim 0.3)$ \\
C8-1-acylcarnitine $\mathrm{mmol} / \mathrm{L}$ & $0.15 \pm 0.09(\sim 0.3)$ \\
C10-acylcarnitine $\mathrm{mmol} / \mathrm{L}$ & $0.12 \pm 0.07(\sim 0.25)$ \\
C12-acylcarnitine $\mathrm{mmol} / \mathrm{L}$ & $0.05 \pm 0.03(\sim 0.3)$ \\
C12-1-acylcarnitine $\mathrm{mmol} / \mathrm{L}$ & $0.12 \pm 0.05(\sim 0.3)$ \\
C14-1-acylcarnitine $\mathrm{mmol} / \mathrm{L}$ & $0.10 \pm 0.04(\sim 0.3)$ \\
C16-acylcarnitine $\mathrm{mmol} / \mathrm{L}$ & $2.0 \pm 0.9(0.4 \sim 3.0)$ \\
C18-acylcarnitine $\mathrm{mmol} / \mathrm{L}$ & $0.76 \pm 0.54(\sim 2.0)$ \\
C18-1-acylcarnitine $\mathrm{mmol} / \mathrm{L}$ & $2.8 \pm 1.4(\sim 2.8)$ \\
C18-2-acylcarnitine $\mathrm{mmol} / \mathrm{L}$ & $0.57 \pm 0.30(\sim 0.8)$ \\
\hline
\end{tabular}

univariate analyses, $\mathrm{C} 0$ or $\mathrm{C} 3$ levels were significantly correlated with age and Child-Pugh score, C2 or C18-1 levels were significantly correlated with only Child-Pugh score, and C16 level was significantly correlated with eGFR and Child-Pugh score (Table 4).

There was no significant difference in each acylcarnitine level by etiology of liver cirrhosis. In multivariate analyses, C0 level was significantly associated with BMI, age, and Child-Pugh score whereas $\mathrm{C} 2$ level was significantly associated with BMI and Child-Pugh score. C16

Table 3 The correlation with Child-Pugh score and carnitine fraction level

\begin{tabular}{lll}
\hline & Correlation Coefficient & $P$-value \\
\hline C0 (Free carnitine) & 0.476 & 0.001 \\
C2-acylcarnitine & 0.607 & 0.001 \\
C3-acylcarnitine & 0.276 & 0.043 \\
C4-acylcarnitine & 0.357 & 0.009 \\
C5-acylcarnitine & 0.114 & 0.413 \\
C6-acylcarnitine & 0.294 & 0.031 \\
C8-acylcarnitine & 0.139 & 0.319 \\
C8-1-acylcarnitine & -0.086 & 0.534 \\
C10-acylcarnitine & 0.356 & 0.008 \\
C12-acylcarnitine & 0.414 & 0.002 \\
C12-1-acylcarnitine & 0.486 & 0.001 \\
C14-1-acylcarnitine & 0.495 & 0.001 \\
C16-acylcarnitine & 0.379 & 0.005 \\
C18-acylcarnitine & 0.162 & 0.246 \\
C18-1-acylcarnitine & 0.636 & 0.001 \\
C18-2-acylcarnitine & 0.571 & 0.001 \\
\hline
\end{tabular}

and C18-1 levels were significantly associated with Child-Pugh score and eGFR.

\section{Questionnaire data associated with carnitine fractions}

CSS scores were significantly associated with the levels of $\mathrm{C} 0, \mathrm{C} 2, \mathrm{C} 3, \mathrm{C} 16$, and C18-1. Scores on sleep questionnaires, such as the PSQI and ESS, were not significantly correlated with levels of any carnitine fraction. On the SF-36 questionnaire, the physical component summary score was significantly associated with the levels of $\mathrm{C} 0, \mathrm{C} 2$, and $\mathrm{C} 18-1$; however, the score of the mental component summary showed no significant correlation with any carnitine fraction level (Table 5).

\section{Discussion}

In this study, we showed that levels of $\mathrm{C} 0$ and acylcarnitines in blood are significantly associated with disease measures in liver cirrhotic patients. The blood levels of most carnitine fractions showed a significant correlation with Child-Pugh score. This result is contrary to a previous study [4]. Shiraki et al. reported that Child-Pugh score was not significantly correlated with total carnitine, free carnitine, or acylcarnitine in cirrhotic patients [4]. The discrepancy may be due to differing methods for measuring carnitine. Shiraki et al. used the enzyme cycling method, which is not able to measure each acylcarnitines, whereas we used the LC-MS/MS method, which can accurately measure each carnitine fraction in blood.

Carnitine dynamics in cytoplasm via carnitinePalmitoyl-Transferase-1 (CPT-1) is involved in the lipid metabolism in the liver $[1,2]$. In this study, we measured carnitine profiling in dried blood sample. Although dried blood sample contains a red blood cell, plasma does not contain the red blood cell component, which means that the measurement in dried blood cell reflects carnitine profiling in cell cytoplasm.

Cirrhosis patients are known to be in a metabolic state similar to starvation [12]. In this study, all patients' blood was sampled $12 \mathrm{~h}$ after dinner. This corresponds to $48 \mathrm{~h}$ of starvation in normal subjects [13]. We found the $\mathrm{C} 2$ acylcarnitine, which is the major carnitine fraction upregulated in starvation [14], was elevated in cirrhosis patients. Changes in lipid metabolism and branched-chain amino acid metabolism are known to occur in patients with advanced cirrhosis [15]. These metabolic changes in advanced cirrhosis patients may involve elevated levels of certain carnitine fractions.

In this study, their diets and dairy activities were variable. With advanced cirrhosis levels, patients tend to receive a restricted diet and dairy activities. These factors can influence on levels of carnitine fractions.

The free carnitine and short-chain acylcarnitines were within standard reference ranges in the majority of liver 


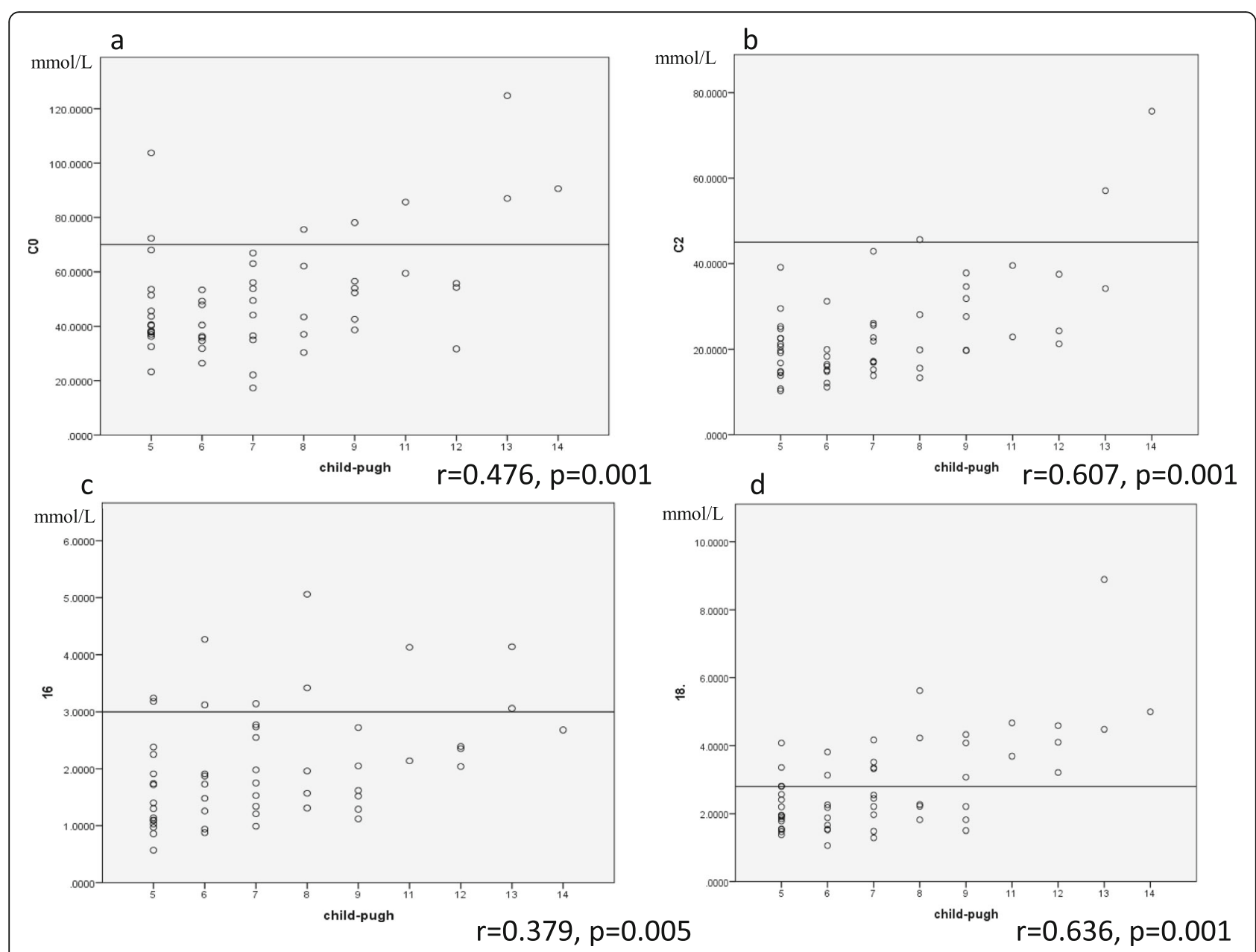

Fig. 1 The Child-Pugh score is significantly correlated with the blood level of C0 (a), C2 (b), C-16 (c), and C18-1 (d). r= correlation coefficient. Each reference quantity is shown as a transverse line

cirrhosis patients; however, long-chain acylcarnitines, such as $\mathrm{C} 16$ and $\mathrm{C} 18-1$, were higher than standard reference ranges in cirrhotic patients and significantly correlated with Child-Pugh score. In the liver, most of the cellular ATP is provided by fatty acid oxidation [16].

Table 4 Association between clinical factors and carnitine fraction level

\begin{tabular}{lllllll}
\hline & & C0 & C2 & C3 & C16 & C18.1 \\
\hline Age & Correlation Coefficient & -0.314 & -0.239 & -0.386 & -0.258 & -0.221 \\
& P-value & 0.021 & .0082 & 0.004 & 0.059 & 0.108 \\
BMI & Correlation Coefficient & -0.243 & -0.237 & -0.148 & -0.106 & -0.131 \\
& P-value & 0.079 & 0.087 & 0.290 & 0.448 & 0.351 \\
eGFR & Correlation Coefficient & -0.054 & -0.138 & 0.107 & $0.282^{*}$ & 0.012 \\
& P-value & 0.700 & 0.321 & 0.442 & 0.039 & 0.931 \\
CPS & Correlation Coefficient & 0.476 & 0.607 & .0276 & 0.379 & 0.636 \\
& P-value & 0.001 & 0.001 & 0.043 & 0.005 & 0.001 \\
\hline
\end{tabular}

Abbreviations: $B M I$ body mass index; eGFR estimated glomerular filtration rate; CPS Child-Pugh score
Long-chain acylcarnitine has an important role in shuttling long-chain fatty acids into mitochondria [17]. Accumulation of long-chain acylcarnitine suggests that more fatty acids can enter mitochondria [18]. The predominant fuel for ATP production shifts from glucose to lipid oxidation in patients with liver cirrhosis. In advanced liver disease, blood free fatty acid levels increase. Long-chain acylcarnitine levels in patients with diabetes showed a significant correlation with free fatty acid [5]. A similar mechanism may be occurring in liver cirrhosis patients. The increased level of long-chain acylcarnitine could be the marker of nutrition status in patient with advanced liver cirrhosis.

Some carnitine fractions levels are associated with age, BMI, renal function. These factors are affected by muscle volume. Carnitine is distributed skeletal muscle. These results may reflect muscle mass volume.

Neither sleep disturbance by the ESS and PSQI nor mental summary scores, assessed by the SF-36 questionnaire, showed any correlation with carnitine fraction levels. On 
Table 5 The correlation with questionnaire data and carnitine fraction level

\begin{tabular}{|c|c|c|c|c|c|c|c|}
\hline & & & $\mathrm{CO}$ & $\mathrm{C} 2$ & $\mathrm{C} 3$ & $\mathrm{C} 16$ & C18.1 \\
\hline \multirow[t]{2}{*}{ CSS } & & Correlation Coefficient & $.314^{*}$ & $.286^{*}$ & $.281^{*}$ & $.379^{* *}$ & $.336^{*}$ \\
\hline & & $P$ value & .026 & .044 & .048 & .007 & .017 \\
\hline \multirow[t]{2}{*}{ PSQI } & & Correlation Coefficient & .148 & .086 & .166 & .048 & .188 \\
\hline & & $P$ value & .312 & .557 & .253 & .741 & .195 \\
\hline \multirow[t]{2}{*}{ ESS } & & Correlation Coefficient & -.075 & .033 & .114 & .141 & .036 \\
\hline & & $P$ value & .611 & .822 & .434 & .334 & .807 \\
\hline \multirow[t]{6}{*}{ SF-36 } & PCS & Correlation Coefficient & $-.355^{*}$ & $-.486^{* *}$ & -.083 & -.262 & $-.403^{* *}$ \\
\hline & & $P$ value & .016 & .001 & .583 & .078 & .005 \\
\hline & MCS & Correlation Coefficient & -.171 & -.191 & -.179 & -.116 & -.207 \\
\hline & & $P$ value & .255 & .203 & .233 & .443 & .168 \\
\hline & RCS & Correlation Coefficient & -.100 & -.175 & -.157 & -.169 & -.243 \\
\hline & & $P$ value & .508 & .246 & .297 & .262 & .103 \\
\hline
\end{tabular}

Abbreviations: CSS cirrhosis-related symptom scores; PSQI Pittsburgh Sleep Quality Index; ESS Epworth Sleepiness Scale; SF-36, 36-item short-form health survey; health; PCS Physical component summary; MCS Mental component summary; RCS Role/Social component summary

the other hand, liver cirrhosis-related symptom scores (CSS questionnaire) and the physical component summary score on the SF-36 were significantly associated with the levels of carnitine fractions. These results indicate that carnitine fractions can be associated with physical symptoms in cirrhotic patients. Liver cirrhosis patients display various symptoms of energy shortage. Supplementation with carnitine is effective for improving symptoms, such as general fatigue, muscle cramps, and hepatic encephalopathy $[4,7,18$, 19]. Carnitine fraction can be the marker of the supplementation with carnitine.

Zhou et al. reported that long-chain carnitines, such as C16 and C18, accumulated to varying degrees in different liver diseases: with the lowest accumulation in chronic hepatitis, then liver cirrhosis, and the highest accumulation in HCC [8]. In our study, multivariate analysis demonstrated that these long-chain carnitines had no significant association with liver cirrhosis or HCC. This difference may be due to the relatively good liver function in HCC patients in this study.

This study has some limitations. First, this study was a small, retrospective study. Additional studies with larger sample sizes are needed to validate this study. Second, this study only enrolled liver cirrhosis patients. Ideally, we would compare liver cirrhosis patients with control subjects. In addition, this study was a cross-sectional study; therefore, we were not able to assess changes in the levels of carnitine fractions over time.

\section{Conclusion}

Elevated carnitine fraction levels significantly correlated with increasing grade of liver cirrhosis, as quantified by Child-Pugh score. This association was particularly strong for long-chain acylcarnitines. Moreover, carnitine fraction levels were also significantly associated with various subjective physical symptoms in liver cirrhosis patients.

\section{Abbreviations}

BMI: Body mass index; CoA: Coenzyme A; CSS: Cirrhosis-related symptom scores; EGFR: Estimated glomerular filtration rate; ESS: Epworth Sleepiness Scale; HCC: Hepatocellular carcinoma; MS/MS: Tandem mass spectrometry; PSQI: Pittsburgh Sleep Quality Index; SF-36: 36-item short-form health survey

\section{Acknowledgements}

Not applicable.

\section{Authors' contributions}

HM: study concept and design, data collection, data analysis, and writing of the draft manuscript; HK: measurement of the samples and study co-design; $\mathrm{SM}, \mathrm{MF}, \mathrm{RS}$, and $\mathrm{MH}$ : data collection; and $\mathrm{KN}$ : study co-design and review of the manuscript. All authors have approved the final version of the article.

\section{Funding}

No funding was received.

\section{Availability of data and materials}

The datasets during and/or analysed during the current study available from the corresponding author on reasonable request.

\section{Ethics approval and consent to participate}

Written informed consent was obtained from all patients. This study was approved by the ethics committee of Nagasaki University (No, 17022735).

\section{Consent for publication}

Not applicable.

\section{Competing interests}

The authors declare that they have no competing interests.

\section{Author details}

${ }^{1}$ Department of Gastroenterology and Hepatology, Nagasaki University Graduate School of Biomedical Sciences, 1-7-1 Sakamoto, Nagasaki 852-8501, Japan. ${ }^{2}$ Department of Pediatrics, Shimane University Faculty of Medicine, 89-1 Enya, Izumo, Shimane 693-8501, Japan. 
Received: 27 September 2019 Accepted: 11 February 2020

Published online: 19 February 2020

\section{References}

1. Fritz IB. Carnitine and its role in fatty acid metabolism. Adv Lipid Res. 1963;1: 285-334.

2. Tanphaichitr V, Leelahagul P. Carnitine metabolism and human carnitine deficiency. Nutrition. 1993;9(3):246-54.

3. Flanagan JL, Simmons PA, Vehige J, Willcox MD, Garrett Q. Role of carnitine in disease. Nutrition metabolism. 2010;7:30. https://doi.org/10.1186/17437075-7-30.

4. Shiraki M, Shimizu M, Moriwaki H, Okita K, Koike K. Carnitine dynamics and their effects on hyperammonemia in cirrhotic Japanese patients. Hepatol Res. 2017;47(4):321-7. https://doi.org/10.1111/hepr.12750.

5. Mihalik SJ, Goodpaster BH, Kelley DE, Chace DH, Vockley J, Toledo FG, et al. Increased levels of plasma acylcarnitines in obesity and type 2 diabetes and identification of a marker of glucolipotoxicity. Obesity (Silver Spring). 2010; 18(9):1695-700. https://doi.org/10.1038/oby.2009.510.

6. Kamei Y, Kamei D, Tsuchiya K, Mineshima M, Nitta K. Association between 4year all-cause mortality and carnitine profile in maintenance hemodialysis patients. PLoS One. 2018;13:e0201591.

7. Nakanishi H, Kurosaki M, Tsuchiya K, Nakakuki N, Takada H, Matsuda S, et al. Lcarnitine reduces muscle cramps in patients with cirrhosis. Clin Gastroenterol Hepatol. 2015;13(8):1540-3. https://doi.org/10.1016/j.cgh.2014.12.005.

8. Zhou L, Wang Q, Yin P, Xing W, Wu Z, Chen S, et al. Serum metabolomics reveals the deregulation of fatty acids metabolism in hepatocellular carcinoma and chronic liver diseases. Anal Bioanal Chem. 2012;403(1):20313. https://doi.org/10.1007/s00216-012-5782-4.

9. Yoshimura E, Ichikawa T, Miyaaki H, Taura N, Miuma S, Shibata H, et al. Screening for minimal hepatic encephalopathy in patients with cirrhosis by cirrhosis-related symptoms and a history of overt hepatic encephalopathy. Biomedical reports. 2016;5(2):193-8. https://doi.org/10.3892/br.2016.702.

10. Johns MW. A new method for measuring daytime sleepiness: the Epworth sleepiness scale. Sleep. 1991;14(6):540-5. https://doi.org/10.1093/sleep/14.6.540.

11. Doi Y, Minowa M, Uchiyama M, Okawa M, Kim K, Shibui K, et al. Psychometric assessment of subjective sleep quality using the Japanese version of the Pittsburgh sleep quality index (PSQI-J) in psychiatric disordered and control subjects. Psychiatry Res. 2000;97(2-3):165-72.

12. Owen OE, Reichle FA, Mozzoli MA, Kreulen T, Patel MS, Elfenbein IB, et al. Hepatic, gut, and renal substrate flux rates in patients with hepatic cirrhosis. J Clin Invest. 1981;68(1):240-52.

13. Hoppel CL, Genuth SM. Carnitine metabolism in normal-weight and obese human subjects during fasting. Am J Phys. 1980;238(5):E409-15. https://doi. org/10.1152/ajpendo.1980.238.5.E409.

14. Paul HS, Adibi SA. Effect of carnitine on branched-chain amino acid oxidation by liver and skeletal muscle. Am J Phys. 1978;234(5):E494-9. https://doi.org/10.1152/ajpendo.1978.234.5.E494.

15. Eaton S, Bartlett K, Pourfarzam M. Mammalian mitochondrial beta-oxidation. Biochem J. 1996;320(Pt 2):345-57. https://doi.org/10.1042/bj3200345.

16. Longo N. Amat di san Filippo C, Pasquali M. disorders of carnitine transport and the carnitine cycle. Am J Med Genet C: Semin Med Genet. 2006; 142C(2):77-85. https://doi.org/10.1002/ajmg.c.30087.

17. Seelaender MC, Curi R, Colquhoun A, Williams JF, Zammitt VA. Carnitine palmitoyltransferase II activity is decreased in liver mitochondria of cachectic rats bearing the Walker 256 carcinosarcoma: effect of indomethacin treatment. Biochem Mol Biol Int. 1998:44(1):185-93.

18. Malaguarnera M, Pistone G, Astuto M, Dell'Arte S, Finocchiaro G, Lo Giudice $E$, et al. L-Carnitine in the treatment of mild or moderate hepatic encephalopathy. Dig Dis. 2003;21(3):271-5. https://doi.org/10.1159/ 000073347.

19. Malaguarnera M, Pistone G, Elvira R, Leotta C, Scarpello L, Liborio R. Effects of Lcarnitine in patients with hepatic encephalopathy. World J Gastroenterol. 2005; 11(45):7197-202. https://doi.org/10.3748/wjg.v11.i45.7197.

\section{Publisher's Note}

Springer Nature remains neutral with regard to jurisdictional claims in published maps and institutional affiliations.

\section{Ready to submit your research? Choose BMC and benefit from:}

- fast, convenient online submission

- thorough peer review by experienced researchers in your field

- rapid publication on acceptance

- support for research data, including large and complex data types

- gold Open Access which fosters wider collaboration and increased citations

- maximum visibility for your research: over $100 \mathrm{M}$ website views per year

At BMC, research is always in progress.

Learn more biomedcentral.com/submissions 